\title{
Two-sided Bounds for Ruin Probability under Constant Interest Force
}

\author{
D.G. Konstantinides*, Q.H. Tang ${ }^{* *}$, and G.Sh. Tsitsiashvili*** \\ * Department of Statistics and Actuarial Science, University of the Aegean, \\ Samos, 83200 Greece \\ email: konstant@aegean.gr \\ **University of Amsterdam, Department of Quantitative Economics \\ Roetersstraat 11, 1018 WB, Amsterdam, The Netherlands \\ email:tangqihe@263.net \\ *** Institute of Applied Mathematics FEB RAS \\ Radio str. 7, Vladivostok, 690041 Russia \\ email:guram@iam-mail.febras.ru \\ Received October 14, 2002
}

In this paper we investigate the ruin probability in the classical risk model under a positive constant interest force. We restrict ourselves to the case where the claim size is heavy-tailed, i.e. the equilibrium distribution function (e.d.f.) of the claim size belongs to a wide subclass (denoted $\mathcal{A}$ ) of subexponential distributions. Two-sided estimates for the ruin probability are developed by reduction from the classical model without interest force. Discussions on the class $\mathcal{A}$ are given.

The classical risk model with homogenous Poisson arrival process, constant premium rate and constant interest force has been investigated by many authors such as Sundt \& Teugels (1995, 1997), Asmussen (1998), Kluppelberg \& Stadtmuller (1998) and Kalashnikov \& Konstantinides (2000). In the present work, we propose two-sided bounds for the ruin probability in this model. The well-known inequalities in the classical risk model without interest force enable us to derive accurate two-sided estimates. The idea of the reduction is not new, but only recently this method became effective as the necessary tools has been accumulated. This approach is also applicable to the investigation of the convergence rate of the ruin probability approximations (see Kalashnikov \& Tsitsiashvili $(1999,2000)$ and Mikosch \& Nagaev (2000)).

We assume that the claim sizes, $\left(Z_{k}\right)_{k \geq 1}$, form a sequence of i.i.d. non-negative r.v.'s, with a common d.f. $B(x)=1-\bar{B}(x)=P\left(Z_{1} \leq x\right), x \geq 0$, and a finite expectation $b$. Throughout this paper, the d.f. $B$ always satisfies $\bar{B}(x)>0$ for all $x \geq 0$. We denote by

$$
F(x)=\frac{1}{b} \int_{0}^{x} \bar{B}(z) d z, \quad x \geq 0
$$

the equilibrium distribution function (e.d.f.) of the d.f. $B$. We assume, as usual, that the claim arrival times constitute a homogeneous Poisson process $(N(t))_{t \geq 0}$, which is independent of $\left(Z_{k}\right)_{k \geq 1}$ and has an intensity $\lambda>0$. Therefore, the compound Poisson process $X(t)=\sum_{k=1}^{N(t)} Z_{k}$ represents the total claim amount accumulated up to time $t \geq 0$, with $X(t)=0$ when $N(t)=0$. We write $\rho=\lambda b$ and let $c$ be the constant gross premium rate which is not necessarily positive. We assume that there exists a constant interest force $\delta>0$ which affects the risk process. Let $u>0$ be the initial surplus of the insurance company, then the total surplus up to time $t$, represented by $U_{\delta}(t)$, satisfies the equation

$$
U_{\delta}(t)=u e^{\delta t}+c \int_{0}^{t} e^{\delta z} d z-\int_{0}^{t} e^{\delta(t-z)} d X(z), \quad t \geq 0
$$


The ultimate ruin probability for this risk process is then defined by

$$
\psi_{\delta}(u)=P\left(\inf _{t \geq 0} U_{\delta}(t)<0 \mid U_{\delta}(0)=u\right), \quad u \geq 0 .
$$

We shall need

$$
G_{\delta}(u)=1-\frac{\psi_{\delta}(u)}{\psi_{\delta}(0)}, \quad k_{\delta}(u)=\int_{u}^{\infty} z d G_{\delta}(z), \quad K_{\delta}=\rho \frac{1-\psi_{\delta}(0)}{\psi_{\delta}(0)} .
$$

See Sundt \& Teugels (1995) for details. These expressions enable us to take the following representation for the ruin probability:

$$
\psi_{\delta}(u)=\frac{\rho}{K_{\delta}+\rho}\left(\frac{k_{\delta}(u)}{u}-\int_{u}^{\infty} k_{\delta}(z) \frac{d z}{z^{2}}\right) .
$$

Further we shall use the following two-sided bounds of $k_{\delta}(u)$ (see Kalashnikov \& Konstantinides (2000))

$$
\frac{\left(\rho+K_{\delta}\right) u}{c+\delta u} \bar{F}(u) \leq k_{\delta}(u) \leq \frac{\left(\rho+K_{\delta}\right)(c-\rho)}{\delta \rho} \frac{\psi_{0}(u)}{1-\psi_{0}(u)} .
$$

The inequalities in (3) will play a critical role in deriving estimates for the ruin probability.

As is done in many recent papers on risk theory, we are interested in heavy-tailed claim sizes. The most important class of heavy-tailed d.f.'s is the subexponential class $\mathcal{S}$. By definition, a d.f. $F$ supported on $[0, \infty)$ belongs to the class $\mathcal{S}$ if and only if for any (or equivalently for some) $n \geq 2$

$$
\lim _{x \rightarrow \infty} \frac{\overline{F^{* n}}(x)}{\bar{F}(x)}=n,
$$

where $F^{* n}$ denotes the $n$-fold convolution of the d.f. $F$. We refer to Embrechts et al. (1997), Goldie \& Kluppelberg (1998), Rolski et al. (1999) and Asmussen (2000) for thorough reviews of the applications of the class $\mathcal{S}$ in insurance and finance.

It is well known that if the e.d.f. $F$ of the claim size belongs to the class $\mathcal{S}$ and the safety loading condition $\rho<c$ holds, then

$$
\psi_{0}(u) \sim \frac{\rho}{c-\rho} \bar{F}(u), \quad u \rightarrow \infty .
$$

See Feller (1971) and Embrechts \& Veraverbeke (1982). This asymptotics, together with inequalities (3), gives the relationship

$$
k_{\delta}(u) \sim \frac{\rho+K_{\delta}}{\delta} \bar{F}(u), \quad u \rightarrow \infty .
$$

Hence, it seems tempting to substitute (5) into (2) on the way to the following asymptotic relationship:

$$
\psi_{\delta}(u) \sim \frac{\lambda}{\delta} \int_{u}^{\infty} \bar{B}(z) \frac{d z}{z}, \quad u \rightarrow \infty .
$$

See Asmussen (1998), Kluppelberg \& Stadtmuller (1998) and Kalashnikov \& Konstantinides (2000) for (6). Definitely, the asymptotics (6) is kept valid under an additional restriction that, for some $v>1$,

$$
\limsup _{x \rightarrow \infty} \frac{\bar{F}(v x)}{\bar{F}(x)}<1 .
$$

Motivated by this background we introduce a new class of subexponential d.f.'s.

Definition. Let $F$ be a d.f. supported on $[0, \infty)$. We say that $F$ belongs to $\mathcal{A}$ if $F \in \mathcal{S}$ and $(7)$ holds for some $v>1$.

ИНФОРМАЦИОННЫЕ ПРОЦЕССЫ ТОМ 2 № 22002 
Theorem 1. In the classical risk model, provided $F \in \mathcal{A}$, the asymptotics 6 is true.

Based on the classical asymptotics (4), Kalashnikov \& Tsitsiashvili $(1999,2000)$ introduced as an auxiliary function the relative error of the approximation (4)

$$
\Delta(u)=\frac{\psi_{0}(u)}{\frac{\rho}{c-\rho} \bar{F}(u)}-1, \quad u \geq 0 .
$$

Obviously, $\Delta(u) \rightarrow 0$ as $u \rightarrow \infty$. Now we look for another auxiliary function which plays a similar role in the present situation. In fact, recalling (6) and the function

$$
D(x)=1-\frac{x}{\bar{F}(x)} \int_{x}^{\infty} \bar{F}(z) \frac{d z}{z^{2}},
$$

we can obtain an asymptotic relationship for the ruin probability $\psi_{\delta}(u)$ that

$$
\psi_{\delta}(u) \sim \frac{\rho}{\delta u} D(u) \bar{F}(u), \quad u \rightarrow \infty .
$$

This urges us to investigate the auxiliary function

$$
\Gamma(u)=\frac{\psi_{\delta}(u)}{\frac{\rho}{\delta u} D(u) \bar{F}(u)}-1, \quad u \geq 0 .
$$

Obviously, $\Gamma(u)$ represents the relative error of the approximation (8).

Now we state the main result of the paper:

Theorem 2. In the classical risk model, if $\rho<c$, then for any $u>0$ we have

$$
\begin{aligned}
& -\frac{1}{D(u)}\left(\frac{c}{c+\delta u}+\frac{\bar{\Delta}(u)+\psi_{0}(u)}{1-\psi_{0}(u)}[1-D(u)]\right) \\
& \quad \leq \Gamma(u) \leq-\frac{c}{c+\delta u}+\frac{1}{D(u)}\left(\frac{c}{c+\delta u}+\frac{\Delta(u)+\psi_{0}(u)}{1-\psi_{0}(u)}\right),
\end{aligned}
$$

where $\bar{\Delta}(u)=\sup _{x \geq u} \Delta(x)$.

\section{REFERENCES}

Asmussen, S., 1998. Subexponential asymptotics for stochastic processes: extremal behaviour, stationary distributions and first passage probabilities. The Annals of Applied Probability 8, 354-374.

Asmussen, S., 2000. Ruin Probabilities. World Scientific, Singapore.

Embrechts, P., Kluppelberg, C., Mikosch, T., 1997. Modelling Extremal Events for Insurance and Finance. Springer, New York.

Embrechts, P., Veraverbeke, N., 1982. Estimates for the probability of ruin with special emphasis on the possibility of large claims. Insurance: Mathematics and Economics 1, 55-72.

Feller, W., 1971. An Introduction to Probability Theory and Its Applications II. Wiley, New York.

Goldie, C.M., Kluppelberg, C., 1998. Subexponential distributions. A practical Guide to Heavy-Tails: Statistical Techniques and Applications. Eds. Adler, R., Feldman, R., Taqqu, M.S. Birkhauser, Boston.

Kalashnikov, V.V., Konstantinides, D., 2000. Ruin under interest force and subexponential claims: a simple treatment. Insurance: Mathematics and Economics 27, 145-149.

Kalashnikov, V.V., Tsitsiashvili, G.Sh., 1999. Tails of waiting times and their bounds. Queueing Systems 32, 257-283. 
Kalashnikov, V.V. Tsitsiashvili, G.Sh., 2000. Tight approximation of basic characteristics of classical and non-classical surplus process. ARCH 00V210(2000-9) 2, 251-293.

Kluppelberg, C., Stadtmuller, U., 1998. Ruin probabilities in the presence of heavy-tails and interest rates. Scandinavian Actuarial Journal, 49-58.

Mikosch, T., Nagaev, A., 2000. Rates in approximations to ruin probabilities for heavy-tailed distributions. Research Institute of Mathematics and Computing Science, Univ. of Groningen, IWI-preprint 2000-5-01.

Rolski T., Schmidli H., Schmidt V., Teugels J., 1999. Stochastic Processes for Insurance and Finance. Wiley, New York.

Sundt, B., Teugels, J.L., 1995. Ruin estimates under interest force. Insurance: Mathematics and Economics 16, 7-22.

Sundt, B., Teugels, J.L., 1997. The adjustment function in ruin estimates under interest force. Insurance: Mathematics and Economics 19, 85-94. 